\title{
Insights from yeast into whether the rapamycin inhibition of heat shock transcription factor (Hsf1) can prevent the Hsf1 activation that results from treatment with an Hsp90 inhibitor
}

\author{
Stefan H. Millson ${ }^{1}$ and Peter W. Piper ${ }^{1}$ \\ ${ }^{1}$ Dept. of Molecular Biology and Biotechnology, University of Sheffield, Western Bank, Sheffield, United Kingdom. \\ Correspondence to: Peter W. Piper, email: peter.piper@sheffield.ac.uk \\ Keywords: heat shock transcription factor; TORC 1; Sch9 protein kinase; rapamycin; Hsp90 inhibitor; FKBP12; Ppt1 protein phospha- \\ tase; yeast \\ Received: March 13, 2014 \\ Accepted: June 6, 2014 \\ Published: June 7, 2014 \\ This is an open-access article distributed under the terms of the Creative Commons Attribution License, which permits unrestricted use, \\ distribution, and reproduction in any medium, provided the original author and source are credited.
}

ABSTRACT:

In human cells TORC1 mTOR (target of rapamycin) protein kinase complex renders heat shock transcription factor 1 (Hsf1) competent for stress activation. In such cells, as well as in yeast, the selective TORC1 inhibitor rapamycin blocks this activation in contrast to Hsp90 inhibitors which potently activate Hsf1. Potentially therefore rapamycin could prevent the Hsf1 activation that frequently compromises the efficiency of Hsp90 inhibitor cancer drugs. Little synergy was found between the effects of rapamycin and the Hsp90 inhibitor radicicol on yeast growth. However certain rapamycin resistance mutations sensitised yeast to Hsp90 inhibitor treatment and an Hsp90 mutation that overactivates Hsf1 sensitised cells to rapamycin. Rapamycin inhibition of the yeast Hsf1 was abolished by this Hsp90 mutation, as well as with the loss of Ppt1, the Hsp90-interacting protein phosphatase that is the ortholog of mammalian PP5. Unexpectedly Hsf1 activation was found to have a requirement for the rapamycin binding immunophilin FKBP12 even in the absence of rapamycin, while TORC1 "bypass" strains revealed that the rapamycin inhibition of yeast Hsf1 is not exerted through two of the major downstream targets of TORC1, the protein phosphatase regulator Tap42 and the protein kinase Sch9 - the latter the ortholog of human S6 protein kinase 1.

Significance: A problem with most of the Hsp90 inhibitor drugs now in cancer clinic trials is that they potently activate Hsf1. This leads to an induction of heat shock proteins, many of which have a "pro-survival" role in that they help protect cells from apopotosis. As the activation of Hsf1 requires TORC1, inhibitors of mTOR kinase could potentially block this activation of Hsf 1 and be of value when used in combination drug therapies with Hsp90 inhibitors. However many of the mechanistic details of the TORC1 regulation of Hsf1, as well as the interplay between cellular resistances to rapamycin and to Hsp90 inhibitors, still remain to be resolved.

\section{INTRODUCTION}

Hsp90, heat shock transcription factor 1 and mTOR kinase - a triage driving cancer progression.

Heat shock protein 90 (Hsp90) provides a molecular chaperone function essential for the conformational maturation, activation and maintenance of proteins essential for sustaining all of the hallmarks of cancer. As such it is now a prime target for drug development, with several Hsp90 inhibitors currently in cancer clinic trials $[1,2]$. Inhibitors of Hsp90 may prove to be most useful for treating those malignancies where the oncogene driver (e.g. HER2, ALK) has a high dependency on Hsp90 or, in situations such as multiple myeloma, where the buffering 
of proteotoxic stress is critical for survival [2]. One factor that often compromises the efficiency of these drugs is their tendency to activate heat shock transcription factor 1 (Hsfl). This leads to the induction of heat shock proteins, many of which have a "pro-survival" role in that they help to protect cancer cells from apopotosis [2,3]. Attention is therefore being directed to developing combination drug therapies that will enable a dual targetting of both Hsf1 and Hsp90 [4]. Like Hsp90, Hsfl is itself a key permissive factor in cancer progression [5] and overexpressed in certain aggressive cancers (e.g. hepatocellular carcinoma [4]). Furthermore Hsfl inhibition may not be toxic to normal tissues since Hsf1 knockout mice are viable [5].

A number of small molecule inhibitors of Hsfl have been identified [6-10]. However the recent discovery that the activation of Hsfl requires mTOR (target of rapamycin) protein kinase [11] indicates the possibility that TOR kinase inhibitors might be as effective as these inhibitors which act directly on Hsfl in blocking the Hsfl activation with by Hsp90 inhibitor drug treatment. Until now, studies that have investigated the combinatorial use of the selective TORC1 inhibitor rapamycin with an Hsp90 inhibitor have not addressed whether the observed effects might be partly due to the rapamycin abrogating the induction of Hsf1 [12-14]. However while this paper was under review, an unbiased drug screen was reported for compounds that could block the Hsfl-directed increases in Hsp70 with Hsp90 inhibition. It revealed that phosphoinositide 3-kinase (PI3K) and mTOR inhibitors could effectively inhibit this increase and potentiate the antitumour efficacy of an Hsp90 inhibitor in many in vivo models [15].

As with both Hsp90 and Hsfl, mTOR is often overactivated in cancer; certain gain of function mutations in the mTOR kinase domain being tumorigenic in animal models $[16,17]$. This protein kinase forms the catalytic subunit of two distinct multiprotein complexes (TORC1/2), complexes which are central to many of the pathways regulating cell growth and proliferation since they act as the integration "hubs" for diverse signalling inputs [16]. Studies of rapamycin, the natural antibiotic identified as the first highly selective inhibitor of TORC1 (see below), either for treating cancer or to promote a healthier, longer life have been well publicized (especially since this agent has been shown to extend lifespan in flies and mice $[18,19])$. Unfortunately the results of the cancer trials of rapamycin and its analogues (rapalogues) have mostly been undistinguished, despite isolated successes. In some cancer cells rapamycin actually promotes oncogenic activity [13], due to an activation of AKT and other signalling molecules of the IGF-1R/IRS1 signalling system which reflects the loss of a negative feedback regulation on IRS-1 and TORC2 [20, 21]. In addition it can increase NFKB activity and upregulate the expression of IGF-1R and HER2 [22]. Rapamycin also has some undesirable side effects, with low dose, long term treatment inducing insulin resistance [23]. Attention is therefore now being directed to the development of inhibitors that will selectively target the catalytic site of mTOR, drugs that will inhibit both TORC1 and TORC2 $[24,25]$ (identifier: www.clinicaltrials.gov). There are indications that such drugs might be highly effective when used in combination with Hsp90 inhibitors. Thus both mTOR inhibitors [13] and Hsp90 inhibitors [1, 2] exert potent antiangiogenic activity, with the expectation that improved antiangiogenic therapies may result from a combined use of these agents. The antiangiogenic properties of the TORC1 inhibitor rapamycin are partly attributable to an inhibition of PI3/AKT signalling in endothelial cells, a process strongly activated by vascular endothelial growth factor (VEGF) [26]. The synergism between rapamycin and Hsp90 inhibitors in cultured breast cancer and multiple myeloma has generally been attributed to key downstream targets of IRS-1 and TORC2 signalling being "clients" of Hsp90 [12, 13]. Indeed the rapamycin-promoted oncogenic activity observed in certain tumors employs a number of signaling components highly dependent on Hsp90 (e.g. IGF-1R, IRS-1, HER2, Erk). It should therefore be abrogated by Hsp90 inhibition. However the discovery that the activation of Hsf1 in human cells requires TORC1 [11], opens the possibility that the results of combinatorial usage of rapamycin with an Hsp90 inhibitor may be partly caused by the rapamycin inhibition of TORC1 abolishing the Hsfl activation with inhibition of Hsp90.

In this study we have employed well-characterised mutant strains of yeast to unravel specific details of the interplay between cellular resistances to rapamycin and an Hsp90 inhibitor; of the TORC1 regulation of Hsfl; of whether the rapamycin inhibition of Hsfl might be overridden by Hsp90 inhibitor treatment; and of how Hsp90 chaperone system defects might impact on the rapamycin inhibition of Hsfl activity.

\section{RESULTS}

\section{Hsp90 inhibitor treatment does not sensitise yeast cells to rapamycin.}

On the basis of current evidence cellular resistances to rapamycin and to Hsp90 inhibitors might be expected to be, at least to a degree, interdependent. Firstly, both in mammalian systems (see Introduction) and in yeast [27] Hsp90 inhibitors activate Hsf1, whereas rapamycin inhibits the activation of Hsf1 [11](see below). Secondly, the activation of Hsfl downregulates TORC1 activity and sensitises yeast to rapamycin [28]. Initially therefore we investigated whether there are any synergistic effects between the inhibitory effects of rapamycin and an Hsp90 inhibitor on yeast growth and whether these might be 
influenced by the loss of the inducible heat shock response.

For this analysis we used two yeast strains (NSY-A, NSY-B; Table 1) which differ in whether they express either a full length or a truncated (residue 1-583) forms of Hsf1. The latter, a form of this transcription factor that lacks the $\mathrm{C}$-terminal activatory domain, provides the Hsfl function needed for growth to $37^{\circ} \mathrm{C}$ yet lacks much of the inducible heat shock response [29, 30]. The cells expressing this truncated (1-583) Hsf1 were appreciably more sensitive to the Hsp90 inhibitor radicicol, consistent with their lower levels of Hsp90 [31]. However they were not sensitised to either rapamycin or caffeine (Fig. 1)(the actions of caffeine in yeast having been largely attributed to its inhibition of TORC1 [32]). Importantly, there was very little synergy between the growth inhibitory effects of the Hsp90 inhibitor radicicol and either rapamycin or caffeine, irrespective of whether the cells were expressing the normal full length Hsf1 or the truncated (1-583) Hsfl (Fig. 1). Therefore, though Hsp90 inhibition normally leads to an activation of Hsfl and the activation of Hsfl in the absence of stress has the potential sensitise cells to rapamycin [28], neither the presence nor absence of a stress-activatable Hsf1 appeared to be causing any appreciable synergy between the inhibitory effects of these two drugs, at least in this model system.

\section{Mutations that confer rapamycin resistance in yeast impact on Hsp90 inhibitor sensitivity.}

The original genetic identification of TOR kinase as the target of rapamycin came through the analysis of mutations causing rapamycin resistance in yeast [33]. Resistance was generated either through the loss of the immunophilin that binds rapamycin (FKBP12) or with alterations to a conserved serine residue within the FKBP12-rapamycin complex binding (FRB) domain of either of the two distinct TOR proteins in yeast (S1972R in Tor1 (the TOR1-1 allele); S1975I in Tor2 (TOR2-1)). Later rapamycin resistance was demonstrated in mammalian cells with the mutation of this conserved serine (S2035) in the single mammalian TOR kinase, mTOR [34, 35]. Not only does the TOR1.1 mutation generate rapamycin resistance, but it also diminishes Tor kinase activity [36]. Extending these studies in yeast we found that both TOR11 and TOR2-1 generated a partial sensitivity to radicicol (Fig. 2a), the most potent natural inhibitor of Hsp90 [37].

Whilst assembly of the TORC1/2 complexes needs the Hsp90 chaperone system [38] no evidence has emerged to date to indicate that the enzymatic activities of these complexes are Hsp90-dependent. Of TORC1/2 subunits, only Raptor ( $\operatorname{Kog} 1$ in yeast) has been shown to interact with Hsp90 [39, 40]. Therefore we investigated whether this effect of TOR1-1 and TOR2-1 sensitising cells to Hsp90 inhibitors (Fig. 2a) might be correlated with an altered regulation of Hsf1. Consistent with this, it was found that these mutations reduced the cellular capacity for induction of a heat shock element (HSE) driven $\beta$-galactosidase gene reporter of Hsfl activity (HSE-lacZ; Fig. 2b). An increased sensitivity to Hsp90 inhibition and dramatically lowered HSE-lacZ induction were also found in the tor $1 \Delta$ mutant (Figs. 2a, 3a,c), a strain which has lowered TORC1 kinase activity due to its lack of the

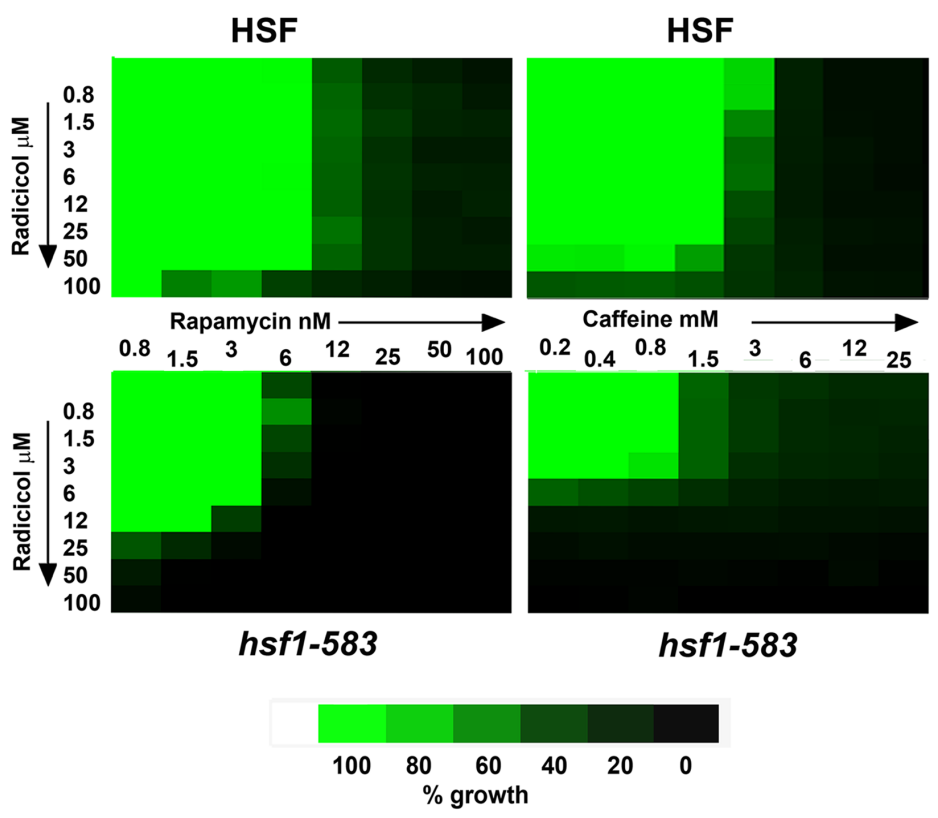

Fig 1: TreeView representation of the growth $\left(46 \mathrm{~h} 28^{\circ} \mathrm{C}\right)$ of strains NSY-A (HSF) and NSY-B (hsf1-583) in the presence of various combinations of radicicol and rapamycin (left) or radicicol and caffeine (right); the data being represented as a percentage of the growth in the absence of drug. 
nonessential Tor1 protein (of the two Tor kinase proteins in Saccharomyces cerevisiae yeast, Tor1 is nonessential and Tor2 essential, both Tor 1 and Tor 2 being capable of forming the catalytic subunit of TORC1 whereas TORC2 contains only Tor2).

Hsf1 activation is lost in yeast, not just with the rapamycin inhibition of TORC1, but also with loss of the rapamycin binding immunophilin FKBP12.

The requirement for TORC1 to render Hsf1 competent for stress activation is clearly apparent from the effects of the selective TORC1 inhibitor rapamycin. We found that in yeast, as in human cells [11], administration of this antibiotic rapidly abolished Hsfl induction (Figs. 2b; 3a,c). Rapamycin must be exerting this effect through its inhibition of TORC1, since it did not inhibit the stress induction of Hsf1 in TOR1-1 and TOR2-1 strains, where the above mutations are causing a genetically dominant

a
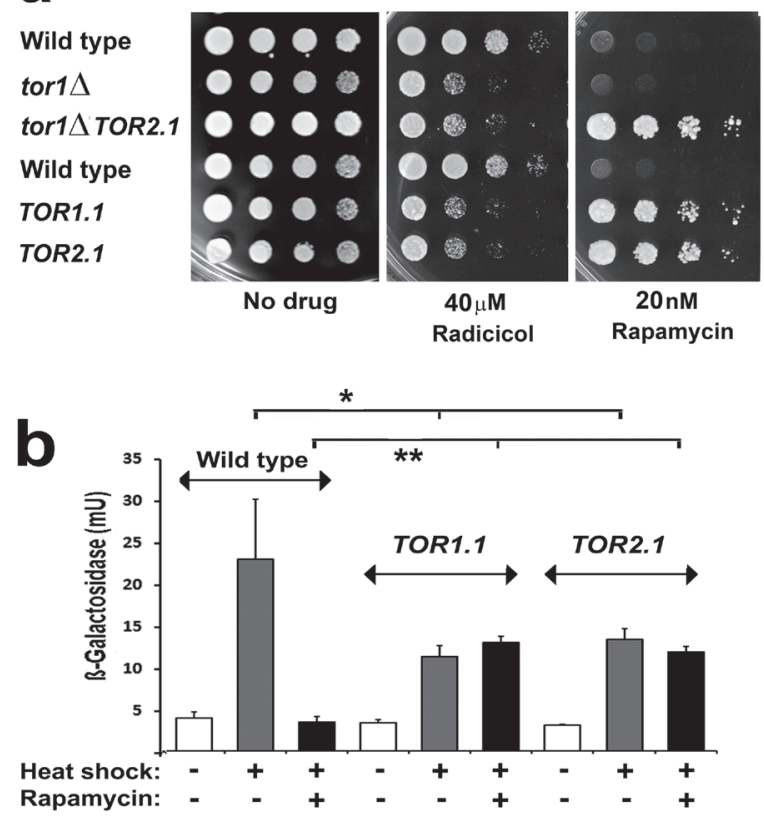

Fig 2 a: Serial dilutions of wild type, tor 1 1 , TOR1-1, TOR2-1 single mutant and tor1 $\triangle$ TOR2-1 double mutant cells of the BY4741 genetic background, pinned onto rich (YPD) medium containing the indicated inhibitor and photographed after $2 \mathrm{~d}$ growth at $28^{\circ} \mathrm{C}$.

b. Measurements of HSE-lacZ reporter gene induction in the wild type, TOR 1-1 and TOR2-1 strains in (a). Cells were treated with or without $100 \mathrm{nM}$ rapamycin for $30 \mathrm{~min}$ at $25^{\circ} \mathrm{C}$, HSElac $Z$ expression being measured after a further $1 \mathrm{~h}$ at $25^{\circ} \mathrm{C}$ or following a $25-39^{\circ} \mathrm{C} 1 \mathrm{~h}$ heat shock (mean and $\mathrm{SD}$ of 8 assays on each culture; two-tailed t-test; $*=\mathrm{p}<0.05 ; * *=\mathrm{p}<0.01)$. loss of the rapamycin inhibition of TORC1 (Fig. 2b).

As described above, rapamycin resistance also arises with the loss of FKBP12, the immunophilin that binds rapamycin. When we investigated HSE-lacZ expression in a strain that lacks this FKBP12 (the fpr $1 \Delta$ mutant;
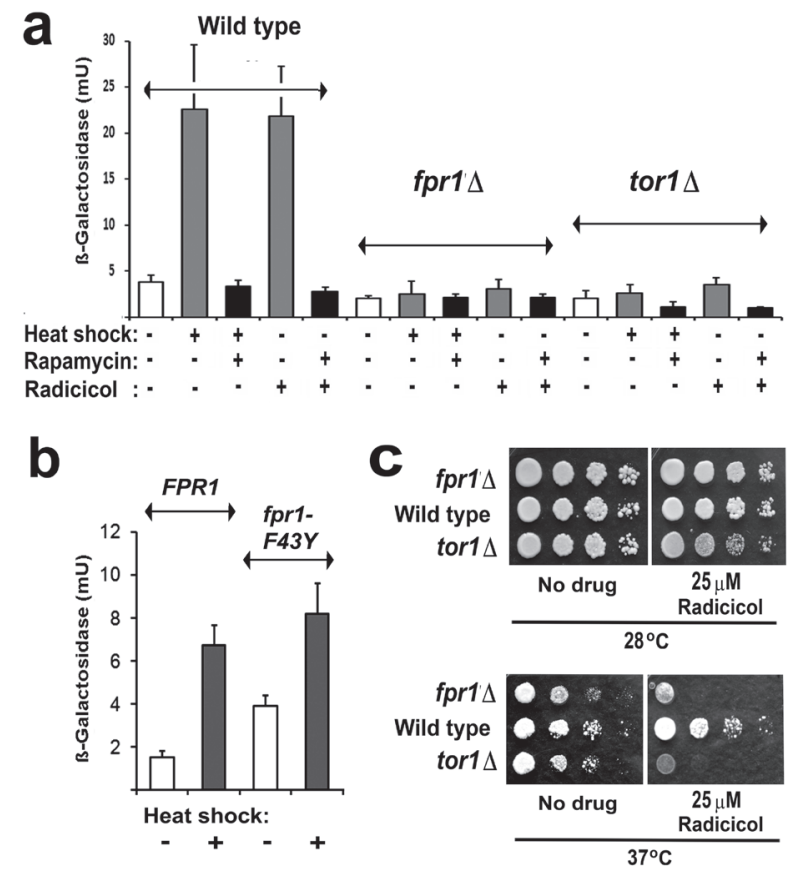

Fig 3 a: Measurements of HSE-lacZ induction in wild type, tor1 $1 \Delta$ and FKBP12-deficient (fpr1A) cells of the BY4741 genetic background. Cells were treated with or without $100 \mathrm{nM}$ rapamycin for $30 \mathrm{~min}$ at $25^{\circ} \mathrm{C}$ prior to $\mathrm{HSE}-\mathrm{lac} Z$ induction, either by heat shock $\left(25-39^{\circ} \mathrm{C}\right.$ for $\left.1 \mathrm{~h}\right)$ or the addition of $100 \mu \mathrm{M}$ radicicol $\left(1 \mathrm{~h} 25^{\circ} \mathrm{C}\right)$. b. The same strains pinned onto rich (YPD) medium with or without radicicol and photographed after $2 \mathrm{~d}$ growth at either $28^{\circ} \mathrm{C}$ or $37^{\circ} \mathrm{C}$. c. HSE-lacZ induction in BY4741fpr $1 \triangle$ mutant cells containing a $L E U 2$ plasmid borne gene for either the wild type (FPRl) or the F43Y mutant (fprlF43Y) FKBP12.

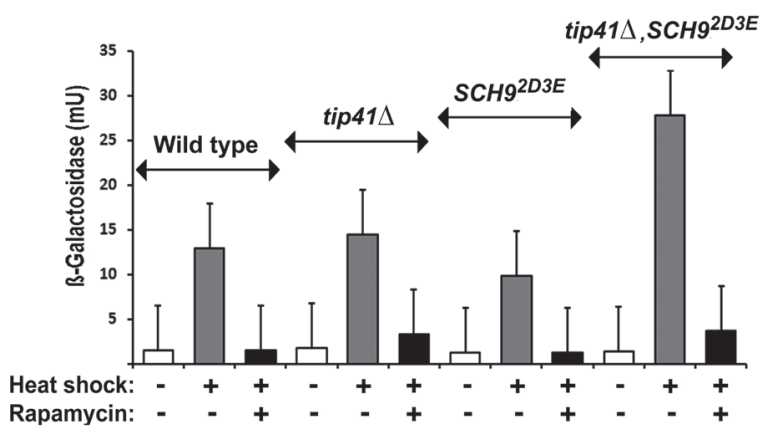

Fig 4: The rapamycin inhibition of HSE-lacZ induction is still apparent in tip41 and $\operatorname{Sch}^{2 \mathrm{D} 3 \mathrm{E}}$ single mutant, also a tip41 $\Delta, \operatorname{Sch} 9^{2 \mathrm{D} 3 \mathrm{E}}$ double mutant, TORC1 "bypass" strains (cells of the TB50a genetic background; Table 1). The conditions of heat shock and rapamycin treatment were as in Figs. 2,3. 
Fig. 3a) we unexpectedly found an almost total absence of stress activation even in the absence of rapamycin. An FPR1 gene-bearing plasmid was able to restore HSE-lacZ activation in this strain (Fig. 3b). Therefore the stress activation of Hsf1 in yeast has a hitherto undiscovered requirement for FKBP12.

Binding of the inhibitory FKBP12/rapamycin complex to TORC1 is known not to require the peptidyl prolyl isomerise activity of the immunophilin FKBP12 [34]. To analyse whether the activation of Hsf1 has a requirement for this peptidyl prolyl isomerase activity we expressed both wild type and F43Y mutant forms of FKBP12 in fpr $1 \Delta$ cells. F43Y corresponds to a mutation which, in the human FKBP12, lowers the peptidyl prolyl isomerise activity 1000-fold [34]. This F43Y mutation did not abolish the capacity of an introduced FPR1 gene to rescue stress activation of Hsfl in fpr $1 \Delta$ cells (Fig. 3b), revealing that FKBP12 does not require its peptidyl prolyl isomerase activity in order to facilitate the activation of Hsfl.

The Hsfl activation defects of tor $1 \Delta$ and fpr $1 \Delta$ mutant cells (Fig. 3a) are one probable reason why both of
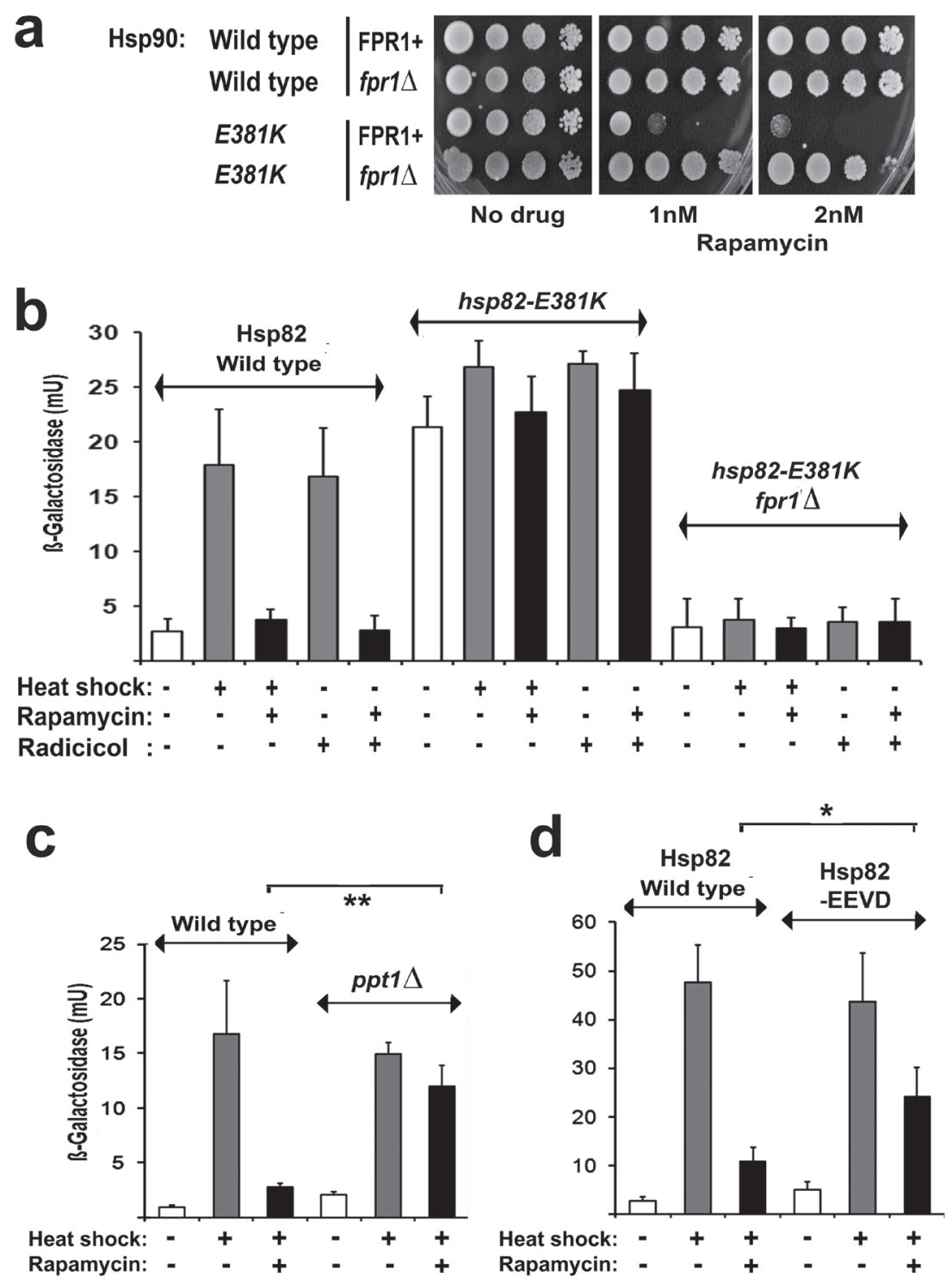

Fig 5: a FPR1+ and fpr $1 \triangle$ cells of the PP30 genetic background expressing either the wild type or the E381K mutant Hsp82 as their sole Hsp90, pinned onto rich (YPD) medium containing the indicated level of rapamycin and photographed after $2 \mathrm{~d}$ growth at $28^{\circ} \mathrm{C}$. b-d Measurements of HSE-lacZ reporter gene expression in: (b) wild type, hsp82-E381K and hsp82-E381K fpr 14 strains of the PP30 genetic background; (c) wild type and ppt1 strains of the BY4741 genetic background; and (d) strains of PP30 genetic background expressing Hsp82 the wild type or the-EEVD mutant Hsp82 as their sole Hsp90. The conditions of heat shock and rapamycin treatment were as in Figs. 2,3. 
Table 1: Strains used in this study

\begin{tabular}{|c|c|c|}
\hline Strain & Genotype & Source \\
\hline W303-1a & $\begin{array}{l}\text { MAT } \alpha ; \text { ade2-1 can1-100 his3-11,15 leu2-3,112 trp1-1 } \\
\text { ura3-1 }\end{array}$ & Euroscarf \\
\hline NSY-A & W303-1a hsf1::LEU2 (pRS314-HSF) & {$[54]$} \\
\hline NSY-B & W303-1a hsf1::LEU2 (pRS314-HSF(1-583)) & {$[54]$} \\
\hline BY4741 & MATa his3- $\Delta 1$ leu2- $\Delta 0$ met15- $\Delta$ ura3- $\Delta 0$ & Euroscarf \\
\hline BY4741 fpr1 $\Delta$ & BY4741 fpr1 $\Delta$ kanMX4 & Research Genetics \\
\hline BY4741 tor $1 \Delta$ & BY4741 tor $1 \Delta \mathrm{kanMX} 4$ & Research Genetics \\
\hline BY4741 TOR1.1 & BY4741 TOR1.1 & This study \\
\hline BY4741 TOR2.1 & BY4741 TOR2.1 & This study \\
\hline BY4741 tor $1 \Delta$ TOR2.1 & BY4741 tor $1 \Delta \mathrm{kanMX} 4 \mathrm{TOR} 2.1$ & This study \\
\hline BY4741 ppt1 & BY4741 ppt1 $\Delta$ kanMX4 & Research Genetics \\
\hline TB50a & MATa; ura3-52, trp1, leu2, his3, rme1 & Robert Loewith \\
\hline AH308 & TB50a SCH9::pRS304 & Alexandre Huber \\
\hline AH333 & TB50a sch92D3E::pRS304 & Alexandre Huber \\
\hline AH386 & TB50a tip41::hphMX4 SCH9::pRS304 & Alexandre Huber \\
\hline AH387 & TB50a tip41::hphMX4 sch92D3E::pRS304 & Alexandre Huber \\
\hline PP30-HSP82 & $\begin{array}{l}\text { MATa trp1-289, leu2-3,112, his3-200, ura3-52, ade2- } \\
\text { 101 oc, lys2-801am, hsc82::kanMX4, hsp82::kanMX4 } \\
\text { [pHSCprom-HSP82 a (LEU2)] }\end{array}$ & {$[27]$} \\
\hline PP30-hsp82(E381K) & PP30 [pHSCprom-hsp82(E381K) ${ }^{\text {a }}$ (LEU2)] & This study \\
\hline PP30-HSP82 fpr1 $\Delta$ & PP30 fpr1 $\Delta$ hphMX4 [pHSCprom-HSP82 a (LEU2)] & This study \\
\hline $\begin{array}{l}\text { PP30-hsp82(E381K) } \\
\text { fpr1 } \Delta\end{array}$ & 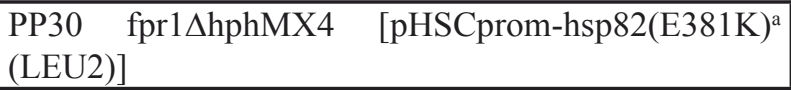 & This study \\
\hline PP30-hsp82(-EEVD) & PP30 [pHSCprom-hsp82(-EEVD) a (LEU2)] & This study \\
\hline
\end{tabular}

a The sole Hsp90 gene in this strain, expressed under HSC82 promoter control and borne on a LEU2 plasmid.

these mutants are slightly temperature sensitive (identifier: www.yeastgenome.org/)(Fig. 3c). Only at higher temperatures did the loss of FKBP12 cause an increased cellular sensitivity to the Hsp90 inhibitor radicicol (the fpr $1 \Delta$ mutant, Fig. 3c).

\section{Rapamycin inhibition of Hsf1 is not exerted through the TORC1 regulation of Tap42 or Sch9, the latter the ortholog of mammalian S6 protein kinase 1.}

In yeast one can address the issue of whether the TORC1 activation of Hsfl is direct, or operates through the known signalling events downstream of TORC1. This model organism can be engineered so that each downstream target phosphorylated by TORC1 is rendered independent of TORC1 activity [41]. Yeast TORC1 is known to directly phosphorylate Tap42 (a regulator of PP2A and PP2A-like protein phosphatases [42]), Sch9 (the budding yeast ortholog of the mammalian S6 protein kinase 1 (S6K1) [41]) and Atg13, a subunit of the Atg1 kinase complex. All three targets are also regulated by other protein kinases (Sch9 by Pkh1/2, functional orthologs of mammalian phosphoinositide-dependent protein kinase 1; $\operatorname{Atg} 13$ by protein kinase A [43]). Tap42 is rendered constitutively active and independent of upstream signals from TORC1 with the loss of its negative regulator, Tip41 [44]. Sch9, the protein kinase whose inactivation causes most of the rapamycin-induced changes to the yeast phosphoproteome [45], is rendered constitutively active and independent of upstream signals from TORC 1 when the amino acids normally phosphorylated by TORC1 at its $\mathrm{C}$-terminus are replaced by acidic residues (the Sch ${ }^{2 \mathrm{D} 3 \mathrm{E}}$ allele [41]).

To determine whether the TORC1 regulation of Hsf1 in yeast (Fig. 2b,c) acts through either Tap42 or Sch9 we investigated how rapamycin affects the stress induction of Hsf1 in TORC1 bypass (tip414, Sch92D3E single mutant and tip $41 \Delta, \mathrm{Sch} 9^{2 \mathrm{D} 3 \mathrm{E}}$ double mutant) strains (Table 1). We were unable to detect any altered sensitivity to Hsp90 inhibitors in such strains (not shown). Hsfl induction was still inhibited by rapamycin in tip $41 \Delta$ cells, lacking the TORC1 regulation of Tap42; Sch9 ${ }^{2 \mathrm{D} 3 \mathrm{E}}$ cells lacking the TORC1 regulation of Sch9; as well as in the tip414, Sch9 ${ }^{2 \mathrm{D} 3 \mathrm{E}}$ double mutant (Fig. 4). Therefore 
the Hsfl inactivation upon rapamycin inhibition of TORC1 (Fig. 2b) does not involve the downregulation of TORC1 signalling through either Tap42 or Sch9. Instead it is possible that TORC1 acts more directly upon Hsf1 in order to render the latter competent for stress activation. It should be noted that a number of the strains studied here (e.g. TOR1-1 and TOR2-1 (Fig. 2b); Sch92D3E (Fig. 4)) are also defective in the rapamycin-mediated phosphorylation of eIF $2 \alpha$, leading to downregulation of protein synthesis [41]. The rapid rapamycin inhibitions of HSE-lacZ expression, measured as loss of $\beta$-galactosidase induction in these experiments (Figs. 2b, 3a,c), are primarily not therefore a reflection of the general downregulation of protein synthesis which normally accompanies the loss of Sch9 activity.

\section{Rapamycin inhibition of Hsf1 can be lost with mutation of the Hsp90 chaperone system.}

Hsp90 inhibitors potently activate Hsfl, due to the inverse correlation between the activity of Hsfl and the activity of the Hsp90 chaperone. The resultant induction of antiapoptotic heat shock proteins is one of the main drawbacks to the use of these drugs in cancer therapy (see Introduction). In yeast, los of this Hsp90 suppression of Hsfl can occur not just through Hsp90 inhibitor treatment [27], but also with mutation to Hsp90 itself (e.g. the hsp82-E381K mutant $[27,46])$ and with defects in certain nonessential cochaperones of the Hsp90 chaperone system [47]. Strain PP30-hsp82(E381K)(Table 1) possess a point mutation in its Hsp90 that causes its Hsf1 to be constitutively hyperactive even in the absence of stress [46] (this Hsfl activation possibly being the reason this strain displays an increased sensitivity to Hsp90 inhibitors [27]). We found that this PP30-hsp82(E381K) also exhibits an extreme, FKBP12 (Fpr1)-dependent hypersensitivity to rapamycin (its growth being inhibited by just $1-2 \mathrm{nM}$ rapamycin; Fig. 5a). This sensitisation to rapamycin can be attributed to its high Hsfl activity, since Hsf1 activation in the absence of heat stress is known to enhance sensitivity to rapamycin [28]. Moreover, unlike the normal Hsf1 activity of PP30-HSP82 cells expressing the wild type Hsp82, this elevated Hsf1 activity of PP30hsp82(E381K) - though still FKBP12-dependent - was not subject to inhibition by rapamycin (Fig. 5b).

We found that rapamycin also did not inhibit the activation of Hsfl in cells that lack the serine/threonine protein phosphatase Ppt1 (the ppt1 $1 \Delta$ mutant, Fig. 5c). Ppt1, the yeast ortholog of mammalian protein phosphatase 5 (PP5), is a dedicated regulator of the Hsp90 chaperone system, its N-terminal tetratricopeptide repeats (TPRs) interacting with the -EEVD motif found at the extreme C-terminus of cytosolic Hsp90s [48]. Finding that this ppt $1 \Delta$ strain displayed a normal HSE-lacZ induction with stress, not inhibited by rapamycin, led us to investigate another strain in which the sole Hsp90 lacks its terminal -EEVD motif (PP30-hsp82(-EEVD); Table 1). In this PP30-hsp82(-EEVD) the HSE-lacZ induction with stress was only partially abolished under our conditions of rapamycin treatment (Fig. 5d). In contrast to hsp82-E381K cells, with their high basal HSE-lacZ expression (Fig. $5 b)$, the ppt $1 \Delta$ mutant and the cells in which the Hsp90 lacks the terminal-EEVD displayed essentially unaltered basal levels of HSE-lacZ expression (Fig. 5) as well as sensitivities to radicicol and rapamycin (not shown).

Together these results provide the first demonstration that the rapamycin inhibition of Hsfl can be lost through Hsp90 mutation or with the loss of an Hsp90 system cochaperone. Rapamycin inhibition of Hsfl is also lost with rapamycin resistance mutations (Fig. 2b), but these act by abolishing the rapamycin/FKBP12 complex binding to TORC1. In contrast the Hsp90 system defects analysed here do not generate rapamycin resistance ( $h s p 82-E 381 \mathrm{~K}$ instead sensitising cells to this antibiotic (Fig. 5a)). It is quite probable therefore that these Hsp90 chaperone defects are preventing the rapamycin inhibition of HSElacZ induction at the level of the Hsp90 suppression of Hsfl activity.

\section{DISCUSSION}

It is important to develop strategies for the inhibition of Hsfl, since this transcription factor is a driver of cancer progression and the Hsfl-directed heat shock response frequently causes a degree of resistance to Hsp90 inhibitor cancer drugs (see Introduction). This study has exploited well characterised mutants of yeast to investigate the rapamycin inhibition of the native Hsfl in this model organism; as well as how this is inhibition might be affected by the inhibition and mutation of the Hsp90 chaperone machine. Both in yeast and in human cells rapamycin and Hsp90 inhibitors have potent, yet opposing, effects on the activity of Hsf1. Rapamycin is an inhibitor of Hsf1 activity (Fig. 2b) [11], while Hsp90 inhibitors are activators of Hsfl. The issue therefore arises of what happens to both growth inhibition and Hsf1 activity when rapamycin is used in combination with an Hsp90 inhibitor.

In this study we found little synergy between the inhibitory effects of rapamycin and the Hsp90 inhibitor radicicol on the growth of yeast, irrespective of whether the cells possessed a stress-inducible form of Hsfl (Fig. 1). This may be due to an overriding effect of rapamycin, which rapidly blocked any Hsfl induction by the Hsp90 inhibitor (Fig. 3a). Such findings contrast with those of a recent study in which mTOR inhibitors were found to potentiate the efficacy of the Hsp90 inhibitor ganetespib in several tumor cell lines [15]. Such differences - yeast versus tumor cells - are perhaps to be expected, especially considering the considerably less complex role of TORC1 in yeast as compared to mammalian systems. 
The novelty of this work is that it has provided the first demonstration that Hsp90 inhibitor resistance can be influenced by mutations causing resistance to rapamycin (Fig. 2a) and, conversely, that rapamycin resistance can be influenced by mutations in the Hsp90 chaperone machine (Fig. 5a). While rapamycin inhibits the activation of Hsf1, it is without any appreciable effect on immediate HSElacZ expression in cells that substantially lack an inducible Hsf1 (e.g. tor $1 \Delta$, fpr $1 \Delta$; Fig. 3a). In yeast this inhibition of Hsf1 is not exerted through either Tap42 or Sch9, major downstream targets phosphorylated by TORC1 (Fig. 4). It may therefore reflect a more direct TORC1 activation of Hsf1. In human cells Hsfl activation is known to require its TORC1-dependent phosphorylation of serine 326 [11]. The phosphorylation of this serine residue is attracting increased attention as a marker of the highly malignant state [49].

This study found that the TOR1.1, TOR2.1 and fpr $1 \Delta$ rapamycin resistance mutations sensitise cells to Hsp90 inhibitor treatment (Fig. 2a, 3c) and abrogate the inducible heat shock response even in the absence of rapamycin (Fig. 2b,3a); that the rapamycin inhibition of Hsfl activation does not involve the downstream TORC1 regulation of Tap42 or Sch9; also that the rapamycin inhibition of Hsfl can be overridden by Hsp90 mutation and with loss of an Hsp90 system cochaperone (Fig. 5). Notably we find that Hsfl activation has a requirement for the rapamycin-binding immunophilin FKBP12, though not the peptidyl prolyl isomerise activity of this FKBP12 (Fig. 3 ). It will be interesting to discover if the Hsfl activation in human cells has a similar requirement for the conserved immunophilin FKBP12.

In mammalian systems, the signalling downstream of TORC1 is considerably more complex than in yeast. However much of it still operates through S6K1 [16]. An extension of these yeast genetic studies therefore has the potential to reveal yet more details of how TORC1 regulates Hsfl, how the inhibition of TORC1 acts to suppress the inducible heat shock response and how this inhibition, in turn, impacts on the Hsp90 suppression of Hsfl activity. Many of the mechanisms uncovered this way are likely to be conserved, yeast to man. Furthermore the human Hsf1 can, by mutation, be rendered functional in yeast whereupon much of its regulation is found to be conserved in this model eukaryote $[50,51]$.

In aging cells and tissues the levels of TORC1 activity are one of the factors driving senescence, with the result that decreases in this activity have the potential to slow aging [52]. In cancer drug therapy the aim is generally a downregulation of mTOR and Hsf1, whereas in healthy aging an increased Hsfl activity should potentially be beneficial in that it will increase chaperone levels, thereby causing the improved proteostasis that could counteract sarcopenia and neurodegenerative disease $[3,10]$. This study has found that the TORC1 regulation of the transient heat shock response of vegetative yeast occurs independently of Sch9 (ortholog of S6K1; Fig. 4). However a decreased activity of S6K, yet an increased activity of Hsfl are increasingly being identified as two factors important for extending the chronological lifespan of model organisms [18, 19]. They are also - at least to a degree - interdependent, Hsf1 being essential for the extension to lifespan when S6K is downregulated in aging Caenorhabditis elegans [53]. Future studies will therefore need to address the issue of whether the TORC1 regulation of Hsfl, studied here in the inducible heat shock response of vegetative yeast cells, differs from how TORC1 might regulate Hsfl in the normal processes of aging.

\section{MATERIALS AND METHODS}

Yeast strains used in this study are listed in Table 1. These were routinely maintained on YPD $(2 \%(\mathrm{w} / \mathrm{v})$ bactopeptone, $1 \%$ yeast extract, $2 \%$ glucose) $1.5 \%$ agar plates. Transformations with a URA3 vector bearing a LacZ gene under heat shock element control (HSE-LacZ) and measurement of levels of $\beta$-galactosidase expression were as previously described [27]. Cultures containing this plasmid were grown on standard defined (SD) minus uracil dropout medium and subjected to stress and/or antibiotic treatment as described in the figure legends. For the study in Fig. 3c strain BY4741 fpr $1 \Delta$ containing this HSE-LacZ vector was transformed with additional YCplac111-based LEU2 vectors bearing either the wild type or F43Y mutant forms of the FPRl gene under native promoter control, then grown on SD minus uracil and leucine prior to measurements of their HSE-LacZ expression.

For drug sensitivity assays, overnight YPD cultures were either serially diluted, then pinned onto YPD agar containing the indicated antibiotic and the plates incubated as indicated in the figure legends; or they were inoculated into complete SD medium $\left(1 \times 10^{5}\right.$ cells $\left./ \mathrm{ml}\right)$, this dilution being used to set up radicicol and rapamycin or radicicol and caffeine dose response matrixes (final volume 0.2 $\mathrm{ml} /$ well) in $96-$ well microtiter plates. After $46 \mathrm{~h}$ growth at $28^{\circ} \mathrm{C}$ the cells were re-suspended by agitation, their absorbance determined at $595 \mathrm{~nm}$ with a Multiskan Ascent platereader with correction for the background from the corresponding medium, and the data quantitatively displayed with color using the program Java Treeview 1.1 .3 (http://jtreeview.sourceforge.net/).

\section{ACKNOWLEDGEMENTS}

We thank K. Morano, J. Heitman, A. Huber, R. Loewith and A. Kumar for providing strains and plasmids; also Yorkshire Cancer Research for supporting this project.

\section{REFERENCES}

1. Trepel J, Mollapour M, Giaccone G and Neckers L. Targeting the dynamic HSP90 complex in cancer. Nature 
reviews Cancer. 2010; 10(8):537-549.

2. Neckers L and Workman P. Hsp90 molecular chaperone inhibitors: are we there yet? Clin Cancer Res. 2012; 18(1):64-76.

3. Piper PW and Millson SH. Mechanisms of Resistance to Hsp90 Inhibitor Drugs: A Complex Mosaic Emerges Pharmaceuticals 2011; 4:1400-1422.

4. Chen Y, Chen J, Loo A, Jaeger S, Bagdasarian L, Yu J, Chung F, Korn J, Ruddy D, Guo R, McLaughlin ME, Feng F, Zhu P, Stegmeier F, Pagliarini R, Porter D, et al. Targeting HSF1 sensitizes cancer cells to HSP90 inhibition. Oncotarget. 2013; 4:816-829.

5. Dai C, Whitesell L, Rogers $\mathrm{AB}$ and Lindquist S. Heat shock factor 1 is a powerful multifaceted modifier of carcinogenesis. Cell. 2007; 130(6):1005-1018.

6. Lee YJ, Erdos G, Hou ZZ, Kim SH, Kim JH, Cho JM and Corry PM. Mechanism of quercetin-induced suppression and delay of heat shock gene expression and thermotolerance development in HT-29 cells. Molecular and cellular biochemistry. 1994; 137(2):141-154.

7. Nagai N, Nakai A and Nagata K. Quercetin suppresses heat shock response by down regulation of HSF1. Biochemical and biophysical research communications. 1995; 208(3):1099-1105.

8. Yokota S, Kitahara M and Nagata K. Benzylidene lactam compound, KNK437, a novel inhibitor of acquisition of thermotolerance and heat shock protein induction in human colon carcinoma cells. Cancer research. 2000; 60(11):29422948.

9. de Billy E, Powers MV, Smith JR and Workman P. Drugging the heat shock factor 1 pathway: exploitation of the critical cancer cell dependence on the guardian of the proteome. Cell cycle (Georgetown, Tex. 2009; 8(23):38063808 .

10. Neef DW, Turski ML and Thiele DJ. Modulation of heat shock transcription factor 1 as a therapeutic target for small molecule intervention in neurodegenerative disease. PLoS biology. 2010; 8(1):e1000291.

11. Chou SD, Prince T, Gong J and Calderwood SK. mTOR is essential for the proteotoxic stress response, HSF1 activation and heat shock protein synthesis. PloS one. 2012; 7(6):e39679.

12. Roforth MM and Tan C. Combination of rapamycin and 17-allylamino-17-demethoxygeldanamycin abrogates Akt activation and potentiates mTOR blockade in breast cancer cells. Anti-cancer drugs. 2008; 19(7):681-688.

13. Stoeltzing O. Dual-targeting of mTOR and HSP90 for cancer therapy: facing oncogenic feed-back-loops and acquired mTOR resistance. Cell cycle (Georgetown, Tex. 2010; 9(11):2051-2052.

14. De Raedt T, Walton Z, Yecies JL, Li D, Chen Y, Malone CF, Maertens O, Jeong SM, Bronson RT, Lebleu V, Kalluri $\mathrm{R}$, Normant E, Haigis MC, Manning BD, Wong KK, Macleod KF, et al. Exploiting cancer cell vulnerabilities to develop a combination therapy for ras-driven tumors. Cancer cell. 2011; 20(3):400-413.

15. Acquaviva J, He S, Sang J, Smith DL, Sequeira M, Zhang C, Bates RC and Proia DA. mTOR Inhibition Potentiates HSP90 Inhibitor Activity via Cessation of HSP Synthesis. Mol Cancer Res. 2014; 12(5):703-713.

16. Dazert E and Hall MN. mTOR signaling in disease. Current opinion in cell biology. 2011; 23(6):744-755.

17. Murugan AK, Alzahrani A and Xing M. Mutations in Critical Domains Confer the Human mTOR Gene Strong Tumorigenicity. The Journal of biological chemistry. 2013; 288(9):6511-6521.

18. Harrison DE, Strong R, Sharp ZD, Nelson JF, Astle CM, Flurkey K, Nadon NL, Wilkinson JE, Frenkel K, Carter CS, Pahor M, Javors MA, Fernandez E and Miller RA. Rapamycin fed late in life extends lifespan in genetically heterogeneous mice. Nature. 2009; 460(7253):392-395.

19. Bjedov I and Partridge L. A longer and healthier life with TOR down-regulation: genetics and drugs. Biochemical Society transactions. 2011; 39(2):460-465.

20. Stoeltzing O, Meric-Bernstam F and Ellis LM. Intracellular signaling in tumor and endothelial cells: The expected and, yet again, the unexpected. Cancer cell. 2006; 10(2):89-91.

21. Rini BI and Atkins MB. Resistance to targeted therapy in renal-cell carcinoma. The lancet oncology. 2009; 10(10):992-1000.

22. Lang SA, Moser C, Fichnter-Feigl S, Schachtschneider P, Hellerbrand C, Schmitz V, Schlitt HJ, Geissler EK and Stoeltzing O. Targeting heat-shock protein 90 improves efficacy of rapamycin in a model of hepatocellular carcinoma in mice. Hepatology. 2009; 49(2):523-532.

23. Lamming DW, Ye L, Katajisto P, Goncalves MD, Saitoh M, Stevens DM, Davis JG, Salmon AB, Richardson A, Ahima RS, Guertin DA, Sabatini DM and Baur JA. Rapamycininduced insulin resistance is mediated by mTORC2 loss and uncoupled from longevity. Science (New York, NY. 2012; 335(6076):1638-1643.

24. Benjamin D, Colombi M, Moroni C and Hall MN. Rapamycin passes the torch: a new generation of mTOR inhibitors. Nature reviews Drug discovery. 2011; 10(11):868-880.

25. Lamming DW, Ye L, Sabatini DM and Baur JA. Rapalogs and mTOR inhibitors as anti-aging therapeutics. The Journal of clinical investigation. 2013; 123(3):980-989.

26. Phung TL, Ziv K, Dabydeen D, Eyiah-Mensah G, Riveros M, Perruzzi C, Sun J, Monahan-Earley RA, Shiojima I, Nagy JA, Lin MI, Walsh K, Dvorak AM, Briscoe DM, Neeman M, Sessa WC, et al. Pathological angiogenesis is induced by sustained Akt signaling and inhibited by rapamycin. Cancer cell. 2006; 10(2):159-170.

27. Piper PW, Millson SH, Mollapour M, Panaretou B, Siligardi G, Pearl LH and Prodromou C. Sensitivity to Hsp90-targeting drugs can arise with mutation to the Hsp90 chaperone, cochaperones and plasma membrane ATP 
binding cassette transporters of yeast. European journal of biochemistry / FEBS. 2003; 270(23):4689-4695.

28. Bandhakavi S, Xie H, O’Callaghan B, Sakurai H, Kim $\mathrm{DH}$ and Griffin TJ. Hsfl activation inhibits rapamycin resistance and TOR signaling in yeast revealed by combined proteomic and genetic analysis. PloS one. 2008; 3(2):e1598.

29. Morano KA, Santoro N, Koch KA and Thiele DJ. A transactivation domain in yeast heat shock transcription factor is essential for cell cycle progression during stress. Molecular and cellular biology. 1999; 19(1):402-411.

30. Millson SH, Truman AW, Rácz A, Hu B, Nuttall J, Mollapour M, Söti C and Piper PW. Expressed as the sole Hsp90 in yeast, the $\alpha$ and $\beta$ isoforms of human Hsp90 differ in their capacities to activate certain client proteins, while only Hsp90 $\beta$ sensitizes cells to the Hsp90 inhibitor radicicol. The FEBS journal. 2007; 274:4453-4463.

31. Millson SH, Nuttall JM, Mollapour M and Piper PW. The Hsp90/Cdc37p chaperone system is a determinant of molybdate resistance in Saccharomyces cerevisiae. Yeast (Chichester, England). 2009; 26(6):339-347.

32. Wanke V, Cameroni E, Uotila A, Piccolis M, Urban J, Loewith $\mathrm{R}$ and De Virgilio C. Caffeine extends yeast lifespan by targeting TORC1. Molecular microbiology. 2008; 69(1):277-285.

33. Heitman J, Movva NR and Hall MN. Targets for cell cycle arrest by the immunosuppressant rapamycin in yeast. Science (New York, NY. 1991; 253(5022):905-909.

34. Lorenz MC and Heitman J. TOR mutations confer rapamycin resistance by preventing interaction with FKBP12-rapamycin. The Journal of biological chemistry. 1995; 270(46):27531-27537.

35. Alarcon CM, Cardenas ME and Heitman J. Mammalian RAFT1 kinase domain provides rapamycin-sensitive TOR function in yeast. Genes \& development. 1996; 10(3):279288.

36. Reinke A, Chen JC, Aronova S and Powers T. Caffeine targets TOR complex I and provides evidence for a regulatory link between the FRB and kinase domains of Torlp. The Journal of biological chemistry. 2006; 281(42):31616-31626.

37. Piper PW and Millson SH. Spotlight on the microbes that produce Hsp90-targetting antibiotics Open biology. 2012; 2(12).

38. Takai H, Xie Y, de Lange $\mathrm{T}$ and Pavletich NP. Tel2 structure and function in the Hsp90-dependent maturation of mTOR and ATR complexes. Genes \& development. 2010; 24(18):2019-2030.

39. Ohji G, Hidayat S, Nakashima A, Tokunaga C, Oshiro N, Yoshino K, Yokono K, Kikkawa U and Yonezawa K. Suppression of the mTOR-raptor signaling pathway by the inhibitor of heat shock protein 90 geldanamycin. Journal of biochemistry. 2006; 139(1):129-135.

40. Delgoffe GM, Kole TP, Cotter RJ and Powell JD. Enhanced interaction between Hsp90 and raptor regulates mTOR signaling upon T cell activation. Mol Immunol. 2009; 46(13):2694-2698.

41. Urban J, Soulard A, Huber A, Lippman S, Mukhopadhyay D, Deloche O, Wanke V, Anrather D, Ammerer G, Riezman H, Broach JR, De Virgilio C, Hall MN and Loewith R. Sch9 is a major target of TORC1 in Saccharomyces cerevisiae. Molecular cell. 2007; 26(5):663-674.

42. Jiang $Y$ and Broach JR. Tor proteins and protein phosphatase $2 \mathrm{~A}$ reciprocally regulate Tap42 in controlling cell growth in yeast. The EMBO journal. 1999; 18(10):2782-2792.

43. Loewith R and Hall MN. Target of rapamycin (TOR) in nutrient signaling and growth control. Genetics. 2011; 189(4):1177-1201.

44. Jacinto E, Guo B, Arndt KT, Schmelzle T and Hall MN. TIP41 interacts with TAP42 and negatively regulates the TOR signaling pathway. Molecular cell. 2001; 8(5):10171026.

45. Huber A, Bodenmiller B, Uotila A, Stahl M, Wanka S, Gerrits B, Aebersold R and Loewith R. Characterization of the rapamycin-sensitive phosphoproteome reveals that Sch9 is a central coordinator of protein synthesis. Genes \& development. 2009; 23(16):1929-1943.

46. Harris N, MacLean M, Hatzianthis K, Panaretou B and Piper PW. Increasing the stress resistance of Saccharomyces cerevisiae, by the overactivation of the heat shock response that results from Hsp90 defects, does not extend replicative life span but can be associated with a slower chronological ageing of nondividing cells. Mol Gen Genomics. 2001; 265:258-263.

47. Duina AA, Kalton HM and Gaber RF. Requirement for Hsp90 and a CyP-40-type cyclophilin in negative regulation of the heat shock response. The Journal of biological chemistry. 1998; 273(30):18974-18978.

48. Vaughan CK, Mollapour M, Smith JR, Truman A, Hu B, Good VM, Panaretou B, Neckers L, Clarke PA, Workman P, Piper PW, Prodromou C and Pearl LH. Hsp90-dependent activation of protein kinases is regulated by chaperonetargeted dephosphorylation of Cdc37. Molecular cell. 2008; 31(6):886-895.

49. de Billy E, Travers J and Workman P. Shock about heat shock in cancer. Oncotarget. 2012; 3(8):741-743.

50. Batista-Nascimento L, Neef DW, Liu PC, RodriguesPousada $\mathrm{C}$ and Thiele DJ. Deciphering human heat shock transcription factor 1 regulation via post-translational modification in yeast. PloS one. 2011; 6(1):e15976.

51. Takemori Y, Enoki Y, Yamamoto N, Fukai Y, Adachi $\mathrm{K}$ and Sakurai H. Mutational analysis of human heatshock transcription factor 1 reveals a regulatory role for oligomerization in DNA-binding specificity. The Biochemical journal. 2009; 424(2):253-261.

52. Blagosklonny MV. Aging: ROS or TOR. Cell cycle (Georgetown, Tex. 2008; 7(21):3344-3354.

53. Seo K, Choi E, Lee D, Jeong DE, Jang SK and Lee SJ. 
Heat shock factor 1 mediates the longevity conferred by inhibition of TOR and insulin/IGF-1 signaling pathways in C. elegans. Aging Cell. 2013; 12(6):1073-1081.

54. Morano KA and Thiele DJ. The Sch9 protein kinase regulates Hsp90 chaperone complex signal transduction activity in vivo. The EMBO journal. 1999; 18(21):59535962. 